\title{
Importância econômica, epidemiologia e controle das intoxicações por plantas no Brasil ${ }^{1}$
}

\author{
Clarice R.M. Pessoa ${ }^{2}$, Rosane M.T. Medeiros² e Franklin Riet-Correa ${ }^{2 *}$
}

\begin{abstract}
Pessoa C.R.M., Medeiros R.M.T. \& Riet-Correa F. 2013 [Economic impact, epidemiology and control poisonous plants in Brazil.] Importância econômica, epidemiologia e controle das intoxicações por plantas no Brasil. Pesquisa Veterinária Brasileira 33(6):752-758. Hospital Veterinário, Universidade Federal de Campina Grande, CSTR, Patos, PB 58700-310, Brazil. E-mail: franklin.riet@pq.cnpq.br

The economic impact, epidemiology and control of plant poisonings in Brazil are reviewed. With the data obtained from diagnostic laboratories from different Brazilian regions, annual losses due to animal deaths are estimated in approximately 820.761 to 1.755.763 cattle, 399.800 to 445.309 sheep, 52.675 to 63.292 goats, and 38.559 horses. In Brazil toxic plants include 131 species within 79 genera, but this number is increasing continuously. However, most economic losses are caused by few plants including Palicourea marcgravii, Amorimia spp., Senecio spp., Pteridium aquilinum, Ateleia glazioviana and Cestrum laevigatum in cattle, Brachiaria spp. in cattle and sheep, Nierembergia veitchii, Mimosa tenuiflora and Ipomoea asarifolia in sheep, swainsonine-containing plants (Ipomoea carnea, Turbina cordata and Sida carpinifolia) in goats, and Brachiaria humidicola and Crotalaria retusa in horses. The main factors that determine the occurrence and frequency of plant poisoning include palatability, hunger, thirst, social facilitation, unawareness of the plant, access to toxic plants, toxic dose, ingestion period, variations in toxicity, and animal resistance/susceptibility. The results obtained in Brazil with the use of biological control, conditioned food aversion, selection of non-toxic varieties of forages, use of resistant animals to poisoning, and techniques to induce resistance are described.
\end{abstract}

INDEX TERMS: Poisonous plants control, economic impact, epidemiology, resistance, conditioned food aversion, biologic control.

RESUMO.- A importância econômica, epidemiologia e controle das intoxicações por plantas em animais domésticos no Brasil são revisadas. Com os dados dos laboratórios de diagnóstico de diferentes regiões do país, as perdas anuais por mortes de animais foram estimadas em 820.761 a 1.755 .763 bovinos, 399.800 a 445.309 ovinos, 52.675 a 63.292 caprinos e 38.559 equinos. No Brasil, atualmente, o número de plantas tóxicas é de 131 espécies e 79 gêneros e aumenta permanentemente. No entanto, a maioria das perdas são causadas por poucas plantas, incluindo Palicourea marcgravii, Amorimia spp., Senecio spp., Pteridium aquilinum, Ateleia glazioviana e Cestrum laevigatum em bovinos, Brachiaria spp em bovinos e ovinos, Nierembergia veitchii,

\footnotetext{
${ }^{1}$ Recebido em 8 de março de 2013.

Aceito para publicação em 25 de março de 2013.

${ }^{2}$ Hospital Veterinário, Centro de Saúde e Tecnologia Rural (CSTR), Universidade Federal de Campina Grande (UFCG), Patos, PB 58700- 000, Brasil. *Autor para correspondência: franklin.pq@cnpq.edu.br
}

Mimosa tenuiflora e Ipomoea asarifolia em ovinos, plantas que contêm swainsonina (Ipomoea carnea, Turbina cordata e Sida carpinifolia) em caprinos e Brachiaria humidicola e Crotalaria retusa em equinos. Os principais fatores epidemiológicos relacionados às intoxicações por plantas incluem palatabilidade, fome, sede, facilitação social, desconhecimento da planta, acesso a plantas tóxicas, dose tóxica, período de ingestão, variações de toxicidade e resistência/ susceptibilidade dos animais às intoxicações. Quanto aos métodos de controle e profilaxia descrevem-se os resultados obtidos no Brasil com métodos recentemente desenvolvidos, incluindo controle biológico, aversão alimentar condicionada, utilização de variedades não tóxicas de forrageiras, utilização de animais resistentes às intoxicações e técnicas de indução de resistência.

TERMOS DE INDEXAÇÃO: Plantas tóxicas, controle de plantas tóxicas, impacto econômico, resistência, epidemiologia, aversão alimentar condicionada, controle biológico. 


\section{INTRODUÇÃO}

No Brasil, devido a grande extensão territorial, na maioria dos estabelecimentos os animais de produção são criados em pastages nativas ou cultivadas, em sistema extensivo ou semiextensivo. Essa característica promove maior acesso dos animais às plantas tóxicas e explica, em parte, o número crescente de registros de surtos de intoxicações por plantas em animais de produção no país. 0 impacto negativo causado pelas intoxicações por plantas na pecuária justifica o desenvolvimento nas últimas décadas de um grande número de pesquisas para caracterizar a epidemiologia e desenvolver tecnologias de controle e profilaxia dessas intoxicações. 0 objetivo desse trabalho é apresentar uma revisão atualizada das perdas econômicas, epidemiologia e controle das intoxicações por plantas no Brasil.

\section{IMPACTO ECONÔMICO DAS INTOXICAÇÕES POR PLANTAS}

As perdas econômicas causadas pelas intoxicações por plantas são difíceis de estimar devido à escassez de dados. No entanto, as perdas causadas pela morte dos animais são facilmente estimadas através da análise dos dados elaborados por laboratórios de diagnóstico veterinário, sobre as causas de mortes dos animais, nas suas respectivas áreas de abrangência.

No Brasil, de uma população de 221.827.299 bovinos (IBGE 2012) pelo menos 5\% (11.091.365) morre anualmente por diferentes causas. Considerando os dados dos laboratórios de diagnóstico de diferentes regiões, entre $7,4 \%$ e $15,83 \%$ dessas mortes (entre 820.761 e 1.755 .763 bovinos) são causadas por plantas tóxicas (Riet-Correa \& Medeiros 2001, Pedroso et al. 2007, Rissi et al. 2007, Casagrande et al. 2008, Assis et al. 2010). A população de equinos no Brasil é de 5.508.546 cabeças (IBGE 2012), levando em consideração uma mortalidade semelhante à de bovinos (5\%), calcula-se que anualmente morrem 275.427 equinos. Considerando que $14 \%$ dos equinos morrem intoxicados por plantas (Assis et al. 2010), estima-se que 38.559 equinos morrem anualmente por essa causa.

O rebanho de ovinos no Brasil é de 17.662.201 cabeças (IBGE 2012). A expectativa de mortalidade anual nesta espécie é de $18,27 \%$ (Lôbo 2002), isso significa uma mortalidade anual de 3.226 .884 ovinos. Considerado os dados de diagnóstico de diferentes Estados (Pedroso et al. 2007, Borelli et al. 2008, Assis et al. 2010) é possível considerar que anualmente morrem de intoxicação por plantas entre $11,46 \%$ e $13,8 \%$ das mortes que ocorrem no rebanho ovino, ou seja de 399.800 a 445.309 animais. Para caprinos a taxa de mortalidade no Brasil é estimada em 8,77\% (Lôbo 2002) e como a população de caprinos no país é de 9.384 .894 animais (IBGE 2012), estima-se que 823.055 caprinos morrem anualmente. Considerando que $6,4 \%$ a $7,69 \%$ destas mortes são causadas por plantas tóxicas (Assis et al. 2010, Pedroso et al. 2007), pode-se estimar que o número de caprinos que morrem por esta causa é de 52.675 a 63.292 .

Os valores sobre perdas reprodutivas causadas por abortos, infertilidade e malformações associadas ao consumo de plantas tóxicas também são raramente obtidos.
Estudos na região semiárida indicam que malformações causadas pela ingestão de Mimosa tenuiflora são frequentes em ruminantes. Considerando que $10 \%$ das mortes neonatais em caprinos (Medeiros et al., 2005) e 23\% em ovinos (Nóbrega et al., 2005) são devido a malformações, estima-se que anualmente morrem por esta causa, na região semiárida 273.120 cabritos e 259.582 cordeiros (Riet-Correa et al. 2007).

\section{PRINCIPAIS PLANTAS TÓXICAS}

No Brasil, o número de plantas conhecidas como tóxicas para ruminantes e equinos aumenta continuamente. Em 1990, Tokarnia et al. (1990) mencionam a existência de 60 espécies tóxicas. Em 2000, eram conhecidas 90 espécies pertencentes a 52 gêneros (Riet-Correa \& Medeiros, 2001). Em 2004 este número aumentou para 113 espécies e 64 gêneros (Riet-Correa et al., 2007); em 2008, 122 espécies e 71 gêneros (Riet-Correa et al. 2009) e, em 2012, 131 espécies e 79 gêneros (dados não publicados).

Apesar das numerosas plantas tóxicas descritas no Brasil, há algumas que são mais importantes, sendo responsáveis pela maioria das perdas econômicas. Senecio spp. é responsável por $50,7 \%$ a $56,14 \%$ das mortes de bovinos causadas por plantas tóxicas no Rio Grande do Sul (Riet-Correa \& Medeiros 2001, Rissi et al. 2007). Em ovinos, na região sul desse mesmo Estado, Nierembergia veitchii representa $77.8 \%$ das mortes causadas por plantas tóxicas (Riet-Correa \& Medeiros 2001). Ateleia glazioviana é, provavelmente, a principal causa de abortos em bovinos em Santa Catarina e norte do Rio Grande do Sul (Riet-Correa et al. 2007). Pteridium aquilinum é a principal planta tóxica de Santa Catarina, responsável pela morte de 7,16\% dos bovinos necropsiados (Gava et al. 2002) e por $44,44 \%$ das mortes causadas por plantas tóxicas em bovinos (Borelli et al. 2008); a planta também é importante no norte do Rio Grande do Sul, Paraná e na região Sudeste. Amorimia (Mascagnia) spp. e Mimosa tenuiflora são, provavelmente, as principais plantas tóxicas da região Nordeste; no entanto, mesmo causando mortalidade baixa, Ipomoea asarifolia é a planta que causa maior número de intoxicações em ruminantes na região Nordeste e na Ilha de Marajó.

Estima-se que as plantas que causam morte súbita são responsáveis por $50 \%$ das mortes por plantas em bovinos no Brasil. Deste grupo, Palicourea marcgravii é a mais importante, causando perdas econômicas severas em todo o país, exceto nos estados da região Sul, no semiárido do Nordeste e no Mato Grosso do Sul. Outras plantas importantes que causam morte súbita em bovinos são Arrabidaea bilabiata e Arrabidaea japurensis na região Norte (Tokarnia et al. 1990). Amorimia spp. são plantas tóxicas importantes nas regiões Sul, Centro-Oeste e Nordeste.

Severas perdas econômicas são causadas pelas intoxicações por Brachiaria spp. em bovinos e ovinos, principalmente nas regiões Centro-Oeste e Sudeste. Nos Estados de Rio de Janeiro e Espírito Santo, a planta tóxica mais importante é Cestrum laevigatum (Tokarnia et al. 2002). Brachiaria humidicola e Crotalaria retusa são as principais plantas tóxicas para equinos no Brasil sendo que $C$. retusa causa 
intoxicações apenas no semiárido brasileiro. As plantas tóxicas mais importantes para caprinos são as que contêm swainsonina: Ipomoea carnea no Nordeste e Ilha de Marajó; Turbina cordata no Nordeste; e Sida carpinifolia no Sul e Sudeste.

\section{EPIDEMIOLOGIA DAS INTOXICAÇÕES POR PLANTAS}

Os fatores identificados no Brasil como desencadeadores ou facilitadores das intoxicações por plantas são mencionados a seguir.

Palatabilidade. A palatabilidade é definida como a aceitação do sabor dos alimentos. Nas pastagens brasileiras há muitas plantas extremamente palatáveis que causam intoxicação, algumas delas são nativas (Palicourea spp.) outras são cultivadas como forrageiras (Trifolium spp. Brachiaria spp. e Vicia spp.) e outras são invasoras (Amaranthus spp.). No entanto, devemos considerar que animais que não ingerem uma determinada planta podem iniciar a ingeri-la pelo mecanismo de facilitação social. Alguns surtos de intoxicação por plantas ocorrem após a aplicação de herbicidas, o que as torna mais palatáveis.

Facilitação social. Este é, também, um fator muito importante para determinar a ingestão de plantas não palatáveis. Algumas plantas, que normalmente não são ingeridas, podem ser ingeridas por alguns animais em condições de carência de forragem e estes gradualmente induzem outros animais da mesma espécie a ingeri-las. Esse tipo de comportamento, denominado facilitação social, tem sido observado com Ipomoea carnea, Turbina cordata e Jatropha ribifolia no Nordeste (Riet-Correa et al. 2011a).

Fome. A produção de substâncias que reduzem a palatabilidade é um mecanismo de defesa das plantas para reduzir a herbivoria (Cheeke \& Shull 1985). No entanto, a fome é um dos fatores que fazem com que o animal ingira plantas de menor palatabilidade. A fome nos rebanhos é resultante de longos períodos de estiagem, invernos rigorosos ou de privação de alimentos por alta lotação ou transporte. A presença de plantas verdes, não palatáveis, em situações de carência de forragem favorece a sua ingestão, como acontece com a Tephrosia cinerea, no Nordeste ou com Senecio spp., no Sul.

Sede. 0 consumo de água após longo período de privação hídrica reduz a capacidade de seleção dos animais, podendo ingerir plantas que anteriormente não ingeriam (Schuster 1978). Este é um fator importante, principalmente após transportes quando os animais podem passar longos períodos sem beber.

Brotação após as primeiras chuvas. Na região semiárida, imediatamente após as primeiras chuvas do período chuvoso ou mesmo durante o período seco, quando ocorrem precipitações escassas, algumas plantas brotam rapidamente enquanto que outras permanecem sem brotar. Isso faz com que haja uma oferta de poucas plantas verdes, entre elas algumas tóxicas como Thiloa glaucocarpa, Mimosa tenuiflora e Turbina cordata.

Desconhecimento. Algumas plantas são ingeridas somente por animais que as desconhecem, por terem sido criados em locais onde não existem as mesmas. A planta mais conhecida que tem esta epidemiologia é a Baccharis coridifolia. Animais adultos criados em regiões onde a planta ocorre não a ingerem, mesmo em condições extremas de fome. No caso de B. coridifolia foi demonstrado que a planta ao ser ingerida em quantidades não tóxicas causa aversão, que é permanente, pelo menos em animais que permanecem em áreas onde a planta ocorre (Almeida et al. 2013).

Acesso às plantas tóxicas. Alguns árvores ou arbustos como Myoporum laetum e muitas plantas cianogênicas (Anadenanthera colubrina var cebil, Prunus spp., Manihot spp. Cnidoscolus quercifolius) normalmente não são consumidas ou são consumidas em quantidades não tóxicas. Quando a planta é cortada, podada ou ramos se quebram com o vento os animais podem ingerir altas doses em pouco tempo. Este fator explica a crença muito difundida no Nordeste de que muitas plantas são mais tóxicas quando murchas. Na verdade, quando cortadas as plantas murcham, mas não é esse fator que determina a intoxicação e sim o fato de que os animais têm acesso a maiores quantidades do que quando a planta está íntegra. Outro exemplo é a exposição das raízes de Marsdenia hilariana, que são mais palatáveis do que as folhas, que pode ocorrer em solos com erosão e quando o terreno é arado para outros fins. Uma situação semelhante ocorre com Nerium oleander, cujas folhas não são consumidas no arbusto, mas sim quando após podas as folhas são administradas aos animais, muitas vezes misturadas com outras plantas que também foram podadas ou cortadas. No Nordeste, nas épocas de estiagem, numerosas intoxicações ocorrem quando os produtores cortam diferentes plantas, incluindo algumas que são tóxicas e normalmente não são ingeridas pelos animais, e as administram aos mesmos, geralmente misturadas a outras plantas e passadas na forrageira.

Dose tóxica. A quantidade da planta necessária para causar intoxicação é muito variável entre as espécies vegetais. Há plantas extremamente tóxicas, como as do gênero Palicourea $(0,50-0,75 \mathrm{~g} / \mathrm{kg})$ ou $B$. coridifolia $(0,25-0,5 \mathrm{~g} / \mathrm{kg}$ no outono). Outras plantas devem ser ingeridas em grandes quantidades como é o caso de Nierembergia veitchii (acima de $440 \mathrm{~g} / \mathrm{kg}$ ) ou causam intoxicação somente quando constituem a totalidade ou a maior parte da dieta, como é o caso de Brachiaria spp. e algumas leguminosas como Trifolium spp. e Vicia spp.

Período de ingestão. Algumas plantas podem causar intoxicações após uma única ingestão, como as plantas cianogênicas, as leguminosas que causam meteorismo e B. coridifolia. Outras têm que ser ingeridas por períodos mais ou menos prolongados como é o caso de Pteridium aquilinum, Nierembergia veitchii e leguminosas que causam estrogenismo.

Variações de toxicidade. Podem existir variações de toxicidade dentro de uma mesma espécie devido a fatores como variabilidade genética, fase de desenvolvimento, tipo de solo, armazenamento e parte da planta que foi consumida. Algumas plantas, como Senecio spp., são mais tóxicas durante a sua fase de crescimento, outras como B. coridifolia são mais tóxicas durante a floração, enquanto que outras apresentam toxicidade somente em determina fase, 
como é o caso de Amaranthus spp. durante a frutificação e Sorghum spp., na fase de brotação. Algumas plantas como Lantana spp. podem apresentar variações de toxicidade entre variedades de uma mesma espécie, ocorrendo toxicidade somente em algumas variedades. Outras como Cestrum parqui e Echium plantagineum, apresentam grandes variações de toxicidade.

Susceptibilidade/Resistência. Há variações marcadas na susceptibilidade dos animais às intoxicações, como espécie, idade, sexo, resistência individual e resistência adquirida. A resistência, seja mediante a seleção de animais resistentes ou mediante a indução de resistência por diferentes técnicas, pode ser empregada no controle de algumas intoxicações.

Transporte. Algumas intoxicações, como a causada por Lantana spp., ocorrem principalmente em animais transportados, enquanto que animais do local não ingerem a planta. Certamente que alguns dos fatores acima mencionados (fome, sede, desconhecimento) são importantes para a ocorrência de intoxicações em animais transportados, mas existem, provavelmente, outros fatores associados ao transporte que influenciam a ocorrência das intoxicações.

\section{CONTROLE E PROFILAXIA DAS INTOXICAÇÕES POR PLANTAS}

A profilaxia e o controle das intoxicações por plantas no Brasil têm se realizado com base no conhecimento da epidemiologia das mesmas. Algumas medidas preventivas incluem: (1) o manejo dos animais e das pastagens, incluindo evitar o pastejo excessivo, utilizar animais de espécies ou idades resistentes a determinadas plantas e evitar colocar animais recentemente transportados com fome ou sede em pastagens contaminadas por plantas tóxicas; (2) a construção de cercas para isolar áreas infestadas por plantas tóxicas; (3) a eliminação das espécies tóxicas pela retirada manual, utilização de herbicidas, roçadas, capinas, aração, queimadas ou pelo pastejo com animais não susceptíveis; (4) a utilização de sementes controladas para evitar a difusão de espécies tóxicas; (5) a confecção de fenos e silagem cuja contaminação por espécies tóxicas seja evitada; e (6) dispor de reservas de forragem para evitar a carência alimentar durante a seca (Riet-Correa et al. 1993, Riet-Correa \& Méndez 2007, Tokarnia et al. 2012).

Considerando que, no Brasil, essas medidas têm apresentado resultados limitados, foi criado um projeto interdisciplinar e interinstitucional para o desenvolvimento de técnicas de controle das intoxicações por plantas, financiado pelo Instituto Nacional de Ciência e Tecnologia (INCT para o Controle de Intoxicações por Plantas). A seguir são mencionadas algumas das tecnologias desenvolvidadas por esse projeto:

Controle integrado da intoxicação por Brachiaria spp. Considerando o estágio e a espécie de Brachiaria, a espécie e a idade dos animais e a possível experiência prévia dos mesmos em pastagens dessa gramínea é possível controlar a intoxicação por Brachiaria spp. Entre os conhecimentos gerados até agora no Brasil sabe-se que: os bovinos são mais resistentes que os ovinos e que animais jovens são mais susceptíveis (Souza et al. 2010, Riet-Correa et al. 2011b); ovinos criados em pastagens de Brachiaria spp. são mais resistentes do que os criados em outras pastagens e possivelmente essa resistência é de origem genética (Castro et al. 2011, Aniz 2008); pelo menos entre búfalos e ovinos há animais resilientes, ou seja apresentam alterações hepáticas sem sinais clínicos (Riet-Correa et al. 2010, Castro et al. 2011, Riet-Correa et al. 2011b). Quanto ao gênero Brachiaria sabe-se que a concentração de saponinas é maior no período de brotação, embora casos de intoxicação já tenham sido descritos em todos os estágios de desenvolvimento. Estima-se que a protodioscina, principal saponina litogênica, quando presente em níveis de $1 \%$ nas pastagens de Brachiaria spp. é tóxica para ovinos oriundos de outras pastagens (Riet-Correa et al. 2011b), mas até o momento não se conhece a concentração de saponinas tóxica para bovinos. $B$. decumbens é a espécie mais tóxica, seguida de $B$. brizantha e $B$. ruziziensis, enquanto que $B$. humidicola, que causa intoxicação quase que exclusivamente em cavalos, é a menos tóxica (Barbosa-Ferreira et al. 2011). A longo prazo, as duas medidas mais eficientes para o controle da mesma deverão ser a utilização de espécies e variedades com baixos teores de saponinas e o pastoreio com animais resistentes.

Controle biológico. Os ovinos apresentam baixa susceptibilidade aos efeitos crônicos dos alcaloides pirrolizidínicos e o pastoreio por ovinos em áreas invadidas por Senecio spp. tem sido uma prática recomendada para o controle da intoxicação. Alguns estudos têm sido conduzidos para determinar as melhores metodologias a serem empregadas no controle biológico de Senecio spp com ovinos. Uma lotação permanente de 0,5 ovinos por hectare (ha) mantém controlada a população de Senecio spp. (Soares et al. 2000) e o pastejo misto com 3 ovinos por ha e 0 a 2,7 bovinos/ ha controlou totalmente a população de $S$. brasiliensis e $S$. madagascariensis em um prazo de dois anos (Bandarra et al. 2012). No Rio Grande do Sul em uma área severamente invadida por Senecio brasiliensis, Senecio heterotrichius e Senecio selloi, uma lotação de oito ovinos/ha controlaram a planta em um período de 6 meses (Schild 2008).

É possível realizar o controle biológico de plantas mediante o uso de fitopatógenos como insetos e outros patógenos de plantas. Senecio jacobeae foi controlado nos Estados Unidos pela liberação de três insetos: Tyria jacobeae, Longitarsus jacobeae e Pegohylemia seneciella (Coombs et al. 1997). Observações das populações de S. brasiliensis em campos nativos revelaram a presença de diversos insetos causando danos em plantas dessa espécie, alimentando-se tanto das folhas como do caule, entre eles principalmente o Phaedon confinis em sua fase larval e adulta. A especificidade de $P$. confinis para plantas de $S$. brasiliensis foi comprovada em testes laboratoriais, demostrando que a alimentação normal, oviposição, sobrevivência e desenvolvimento estão restritos ao $S$. brasiliensis. 0 fato de $P$. confinis ser suficientemente específico para não constituir risco para outras plantas justifica a continuação das pesquisas para utilizar este inseto no controle biológico de Senecio brasiliensis (Milléo et al. 2010).

Após a verificação experimental de que a administra- 
ção diária continuada de doses não tóxicas de sementes de $C$. retusa induz resistência à monocrotalina em ovinos (Anjos et al. 2010), animais sobreviventes a um surto de intoxicação aguda por sementes de C. retusa foram mantidos no local do surto. Em três anos de pastoreio continuado os ovinos extinguiram as plantas de C. retusa da área, demonstrando que a introdução gradual de ovinos em pastos invadidos por $C$. retusa poderá ser utilizada como modelo de controle biológico de Crotalaria spp. (Riet-Correa et al. 2011c).

Aversão alimentar condicionada. Uma técnica empregada no controle das intoxicações por plantas é a aversão alimentar condicionada. Consiste na administração de cloreto de lítio ( $\mathrm{LiCl}$ ) através de sonda ruminal, imediatamente após a ingestão da planta que se quer evitar o consumo. Em experimentos recentes, realizados em caprinos para testar a aversão alimentar condicionada como forma de controle da intoxicação por I. carnea subsp. fistulosa, foi demonstrado que caprinos recém adaptados à ingestão da planta, submetidos ao tratamento aversivo mediante a administração de $\mathrm{LiCl}(200 \mathrm{mg} / \mathrm{kg})$, logo após a ingestão voluntária da planta, não a ingeriram por pelo menos dois anos e oito meses (Oliveira et al., 2010, 2013a). No entanto, se os animais avertidos convivem com animais não avertidos que ingerem a planta, a aversão desaparece rapidamente (Pimentel et al. 2012) e caprinos provenientes de propriedades em que existe a planta, com sinais clínicos da intoxicação, e que têm o hábito de ingerir a planta por longos períodos, não respondem ao tratamento aversivo (Oliveira et al. 2010, 2013a).

Em um estudo realizado com a finalidade de controlar a intoxicação por Turbina cordata em caprinos, em propriedades onde estava ocorrendo a doença, foi demonstrado que a técnica de aversão alimentar, administrando $\mathrm{LiCl}$ $(175 \mathrm{mg} / \mathrm{kg})$, a cada dois meses, aos animais que ingerem a planta espontaneamente, é eficiente e viável para o controle dessa intoxicação (Pimentel et al. 2012). No entanto, para o controle da intoxicação por I. carnea subsp. fistulosa, a técnica impediu a ingestão da planta somente durante a época de chuva, mas não durante a seca, quando há pouca disponibilidade de forragem (Pimentel et al. 2012). T. cordata, diferentemente de I. carnea subsp. fistulosa, é uma planta que desaparece durante a época seca e rebrota após as primeiras chuvas, de forma que, se os animais têm outras forragens à disposição quando a planta está presente, é mais fácil manter a aversão.

Baccharis coridifolia (mio-mio) é uma planta tóxica comum no Rio Grande do Sul que causa aversão natural ao seu próprio consumo, fazendo com que os animais criados em pastagens contendo a planta não a ingiram (Almeida et al. 2009). Em experimentos com bovinos foi comprovado que mio-mio causa aversão eficiente quando administrado $25 \%$ da dose tóxica e se os animais são soltos em pastagens invadidas pela planta pelo menos 24 horas após a ingestão não se intoxicam. Animais soltos antes desse período não estão ainda fortemente avertidos e podem intoxicar-se (Almeida et al. 2013). Trabalhos estão sendo conduzidos para verificar se é possível causar aversão a I. carnea utilizando o mio-mio como aversivo (Adrien et al. 2013). Em outros experimentos tem sido induzida com sucesso aversão condicionada a Amorimia (Mascania) rigida em caprinos (Barbosa et al. 2008) e ovinos (Pacífico da Silva \& Soto-Blanco 2010) e a Palicourea aeneofusca em caprinos (Oliveira et al. 2013b). Experimentos para induzir aversão a Leucaena leucocephala, que é uma planta palatável, induziram redução parcial do consumo, pois os animais continuaram a ingerir a planta em pequenas quantidades (Górniak et al. 2008).

Os resultados de todos estes experimentos mostram que a técnica de aversão condicionada pode ser utilizada no controle e profilaxia da intoxicação de algumas plantas tóxicas. No entanto, o conhecimento das condições epidemiológicas é importante para determinar a forma de aplicação da técnica e o provável sucesso da mesma, que depende da disponibilidade de forragem, conhecimento prévio da planta por parte dos animais e idade dos mesmos.

Resistência às intoxicações por plantas. Este é um fator importante a ser considerado no controle das intoxicações. Já foi mencionada neste trabalho a resistência à intoxicação por Brachiaria spp. Em um experimento com Crotalaria retusa foi demonstrado que doses únicas de sementes de 3-4 g/kg causam intoxicação aguda em ovinos, enquanto que doses diárias repetidas de $2 \mathrm{~g} / \mathrm{kg}$ induzem resistência à doses únicas de até $10 \mathrm{~g} / \mathrm{kg}$. A resistência a intoxicação por $C$. retusa, adquirida pela ingestão de doses não tóxicas, está provavelmente associada a uma adaptação do sistema P450 do fígado (Anjos et al. 2010). Foi demonstrado, também, que animais que adquirem esta resistência a perdem rapidamente, em pouco mais de uma semana (Maia 2013). Uma situação semelhante ocorre com Senecio brasiliensis em ovinos que ingerindo diariamente doses não tóxicas continuadas adquirem resistência a doses tóxicas (Grecco et al. 2012). A ingestão de pequenas doses consecutivas de favas de Enterolobium cotortisiliquum também induz resistência (Tokarnia et al. 1999) e permite que ovinos e caprinos que permanecem em áreas onde ocorre a planta, ingerindo as favas, durante todo o período de frutificação, sem apresentar sinais clínicos (Riet-Correa F., dados não publicados).

Outro tipo de resistência que está sendo estudado é a utilização de bactérias que hidrolisam monofluoroacetato de sódio (MFA) para o controle de intoxicações por plantas com este princípio ativo. Essa resistência está associada, aparentemente, com bactérias que contém dehalogenases que hidrolisam MFA. Em um trabalho recente com o isolamento de bactérias que degradam MFA de rúmen de caprinos no Nordeste, foi sugerido que essas bactérias já existem no rúmen e que alimentação contendo o substrato estimula a sua multiplicação (Camboim et al. 2012) o que poderia explicar o fato que animais mantidos em áreas com Amorimia (Mascagnia) rigida são mais resistentes do que os mantidos em áreas livres da planta (Silva et al. 2008). Também foi demonstrado que a administração de pequenas quantidades de folhas frescas de Amorimia (Mascagnia) septentrionalis (Duarte et. al. 2013) e Palicourea aeneofusca (Oliveira et al. 2013c) induzem resistência parcial em caprinos e que animais que recebem líquido ruminal de animais resistentes adquirem resistência semelhante.

Oliveira et al. (2013c) sugerem que há três possibilida- 
des a serem exploradas para o controle de plantas que contém MFA mediante o aumento da resistência dos animais: 1) a administração repetida, por períodos alternados, de doses não tóxicas da planta; 2) a administração de MFA em doses não tóxicas o que permitiria, também, a proliferação de bactérias que tenham atividade de dehalogenases; e 3) a administração de outro substrato, não tóxico, que estimule a proliferação de bactérias com atividade de dehalogenases. Considerando as dificuldades de administrar doses não tóxicas das plantas e o risco de trabalhar com MFA a terceira possibilidade pareceria a mais adequada.

Agradecimentos.- Este trabalho foi financiado pelo Instituto Nacional de Ciência e Tecnologia (INCT) para o estudo do Controle das Intoxicações por Plantas, processo CNPq 573534/2008-0

\section{REFERÊNCIAS}

Adrien M.L., Riet-Correa G., Oliveira C.A., Pfister J.A., Cook D., Souza E.E.G., Riet-Correa F. \& Schild A.L. 2013. Conditioned aversion to Ipomoea carnea var. fistulosa induced by Baccharis coridifolia in goats. Appl. Anim. Behav. Sci. (In publication)

Almeida M.B., Schild A.L., Brasil N.A., Quevedo P.S., Fiss L., Pfister J.A. \& Riet-Correa F. 2009. Conditioned aversion in sheep induced by Baccharis coridifolia. Appl. Anim. Behav. Sci. 117:197-200.

Almeida M.B., Schild A.L., Pfister J.A., Brasil N.A., Pimentel M., Forster K.M. \& Riet-Correa F. 2013. Methods of inducing conditioned food aversion to Baccharis coridifolia (mio-mio) in cattle. Ciência Rural. (No prelo)

Aniz A.C.M. 2008. Efeito da adaptação ao consumo de Brachiaria decumbens e a existência de resistência ou susceptibilidade individual em ovinos à intoxicação. Monografia, Universidade Federal de Mato Grosso do Sul, Campo Grande, MS. 31p.

Anjos B.L., Nobre V.M.T., Dantas A.F.M., Medeiros R.M.T., Oliveira Neto T., Molyneux R.J. \& Riet-Correa F. 2010. Poisoning of sheep by seeds of Crotalaria retusa: Acquired resistance by continuous administration of low doses. Toxicon 55:28-32.

Assis T.S., Medeiros R.M.T., Riet-Correa F., Galiza G.J.N., Dantas A.F.M. \& Oliveira D.M. 2010. Intoxicações por plantas diagnosticadas em ruminantes e equinos e estimativa das perdas econômicas na Paraíba. Pesq. Vet. Bras. 30(1):13-20.

Bandarra P.M., Oliveira L.G., Dalto A.G., Boabaid F.M., Juffo G., Riet-Correa F., Driemeier D. \& Cruz C.E.F. 2012. Sheep production as a Senecio spp. control tool. Pesq. Vet. Bras. 32(10):1017-1022.

Barbosa R.R., Pacífico da Silva I. \& Soto-Blanco B. 2008. Development of conditioned taste aversion to Mascagnia rigida in goats. Pesq. Vet. Bras. 28(12):571-574.

Barbosa-Ferreira M.B., Brum K.B., Oliveira N.M.R., Valle C.B., Ferreira V.B. N., Garcez V., Riet-Correa F. \& Lemos R.A.A. 2011. Concentração da saponina esteroidal protodioscina em diferentes espécies e cultivares de Brachiaria spp. Vet. Zootec. 18(Supl.3):580-583.

Borelli V., Zandonai A., Furlan F., Traverso S.D. \& Gava A. 2008. Intoxicação por plantas diagnosticadas em bovinos pelo Laboratório de Patologia Animal/CAV/UDESC. Anais $3^{\circ}$ Endivet, Campo Grande, MS, p.59-60.

Camboim E.K.A., Almeida A.P., Tadra-Sfeir M.Z., Garino Junior F., Andrade P.P., McSweeney C.S., Melo M.A. \& Riet-Correa F. 2012. Isolation and identification of sodium fluoroacetate degrading bacteria from caprine rumen in Brazil. Scientif. World J. Article ID 178254, 6p. doi:10.1100/2012/178254.

Casagrande R.A., Mazzocco M.B., Frizon R., Lentz D., Traverso S.D. \& Gava A. 2008. Doenças de bovinos diagnosticadas pelo Laboratório de Patologia Animal CAV/UDESC de janeiro de 2000 a abril de 2008. Anais $3^{\circ}$ Endivet, Campo Grande, MS, p.55-56.

Castro M.B., Santos Jr. H.L., Mustafa V.S., Gracindo C.V., Moscardini A.C.R., Louvandini H., Paludo G.R., Borges J.R.J., Haraguchi M., Ferreira M.B. \& Riet-Correa F. 2011. Brachiaria spp. poisoning in sheep in Brazil: Experimental and epidemiological findings, p.110-117. In: Riet-Correa F., Pfis- ter J., Schild A.L. \& Wierenga T. (Eds), Poisoning by Plants, Mycotoxins and Related Toxins. CAB International, Willingford.

Cheeke P.R. \& Shull L.R. 1985. Natural toxicants in feeds and poisonous plants. AVI Publishing, Westport, CT. 422p.

Coombs E., Mallory-Smith L.C., Burrill R.H., Callihan R., Parker \& Radtke H. 1997. Tansy ragwort, Senecio jacobaea L. Pacific Northwest Extension Publication 175:1-7.

Duarte A.L.L., Medeiros R.M.T., Carvalho F.K.L., Lee S.T., Cook D., Pfister J.A., Costa V.M.M. \& Riet-Correa F. 2013. Induction and transfer of resistance to poisoning by Amorimia (Mascagnia) septentrionalis in goats. J. Appl. Toxicol. (In publication). DOI 10.1002/jat.2860.

Gava A., Neves D.S., Gava D.S.T.M. \& Riet-Correa F. 2002. Braken fern (Pteridium aquilinum) poisoning in cattle in southerm Brazil. Vet. Human Toxicol. 44(6):362-365.

Górniak S., Pfister J., Lanzonia E. \& Raspantini E. 2008. A note on averting goats to a toxic but palatable plant, Leucaena leucocephala. Appl. Anim. Behav. Sci. 111:396-401.

Grecco F.B., Estima-Silva P., Marcolongo-Pereira C., Soares M.P., Raffi M.B. \& Schild A.L. 2012. Intoxicação experimental aguda por Senecio brasiliensis em ovinos e indução de resistência à intoxicação. Pesq. Vet. Bras. 32(9):912-916.

IBGE 2012. Produção da Pecuária Municipal (PPM). Vol.39. Instituto Brasileiro de Geografia e Estatística, Rio de Janeiro-RJ. 63p.

Lôbo R.N.B. 2002. Melhoramento Genético de Caprinos e Ovinos: desafios para o mercado. Embrapa Caprinos, Sobral, CE. 36p.

Maia L.A. 2013. Intoxicação por Crotalaria retusa em pequenos ruminantes. Dissertação de Mestrado, Universidade Federal de Campina Grande, Patos, PB. 56p.

Milléo J., Corrêa J.H., Pena Castro J. \& Pedrosa-Macedo J.H. 2010. Host specificity of Phaedon confinis (Coleoptera: Chrysomelidae) for the biocontrol of Senecio brasiliensis (Asteraceae). Revta Bras. Entomol. 4(2): 61-66.

Medeiros J.M., Tabosa I.M., Simões S.V.D., Nóbrega Júnior J.E., Vasconcelos J.S. \& Riet-Correa F. 2005. Mortalidade perinatal em caprinos no semiárido da Paraíba. Pesq. Vet. Bras. 25(4):201-206.

Nóbrega Júnior J.E., Riet-Correa F., Nóbrega R.S., Medeiros J.M.,Vasconcelos J.S., Simões S.V.D. \& Tabosa I.M. 2005. Mortalidade perinatal de cordeiros no semi-árido da Paraíba. Pesq. Vet. Bras.25(3):171-178.

Oliveira C.A., Duarte M.D., Cerqueira V.D., Riet-correa B., Riet-correa F., Vinhote W.M. \& Riet-Correa G. 2010. Utilização da técnica de aversão alimentar condicionada em caprinos intoxicados naturalmente por Ipomoea carnea subsp. fistulosa. VI Encontro Nacional de Diagnóstico Veterinário, Campo Grande, MS. (CD-ROM)

Oliveira C.A., Riet-Correa G. \& Riet-Correa F. 2013a. Intoxicação por plantas que contêm swainsonina no Brasil. Ciência Rural 43(4):653-661.

Oliveira M.D., Riet-Correa F., Carvalho F.K., Silva G.B., Pereira W.S. \& Medeiros R.M.T. 2013b. Conditioned food aversion to control Palicourea aeneofusca poisoning. J. Appl. An. Behav. Scien. (Submitted)

Oliveira M.D., Riet-Correa F., Carvalho F.K., Silva G.B., Pereira W.S. \& Medeiros R.M.T. 2013c. Indução de resistência à intoxicação por Palicourea aeneofusca (Rubiaceae) mediante administração de doses sucessivas não tóxicas. Pesq. Vet. Bras. 33(6):731-734.

Pacífico da Silva I. \& Soto-Blanco B. 2010. Conditioning taste aversion to Mascagnia rigida (Malpighiaceae) in sheep. Res. Vet. Sci. 88:239-241.

Pedroso P.M.O., Pescador C.A.P., Oliveira E.C., Sonne L., Bandarra P.M., Raymundo D.L. \& Driemeier D. 2007. Intoxicações naturais por plantas em ruminantes diagnosticadas no Setor de Patologia Veterinária da UFRGS no período de 1996-2005. Acta Sci. Vet. 35:213-218.

Pimentel L.A., Maia L.A., Campos E.M., Dantas A.F.M., Medeiros R.M.T., Pfister J.A., Cook D. \& Riet-Correa F. 2012. Aversão alimentar condicionada no controle de surtos de intoxicações por Ipomoea carnea subsp. fistulosa e Turbina cordata. Pesq. Vet. Bras. 32(8):707-714.

Riet-Correa F., Méndez M.C. \& Schild A.L. 1993. Intoxicação por Plantas e Micotoxicoses em Animais Domésticos. Editorial Agropecuária Hemisferio Sur, Montevideo. 340p. 
Riet-Correa F. \& Medeiros R.M.T. 2001. Intoxicações por plantas em ruminantes no Brasil e no Uruguai: importância econômica, controle e riscos para a saúde pública. Pesq. Vet. Bras. 21:38-42.

Riet-Correa F. \& Méndez M.C. 2007. Intoxicações por plantas e micotoxinas, p.99-221. In: Riet-Correa F., Schild A.L., Lemos R.A.A. \& Borges J.R.J. (Eds), Doença de Ruminantes e Eqüídeos. Pallotti, Santa Maria.

Riet-Correa F., Medeiros R.M.T., Tokarnia C.H. \& Döbereiner J. 2007. Toxic plants for livestock in Brazil: economic impact, toxic species, control measures and public health implications, p.2-14. In: Panter K.E., Wierenga T.L., Pfister J.A. (Eds), Poisonous Plants: global research and solutions. CAB International, Wallingford.

Riet-Correa F., Pfister J., Schild A.L., Medeiros R.M. \& Dantas A.F.M. 2009. Poisonings by Plants, Mycotoxins and Related Substances in Brazilian Livestock. Pallotti, Santa Maria. 246p.

Riet-Correa B., Riet-Correa F., Oliveira Junior C.A., Duarte V.C. \& Riet-Correa G. 2010. Alterações histológicas em fígados e linfonodos de búfalos (Bubalus bubalis) em pastagens de Brachiaria spp. Pesq. Vet. Bras. 30(9):705-711.

Riet-Correa F., Bezerra C.W.C. \& Medeiros R.M.T. 2011a. Plantas Tóxicas do Nordeste. Sociedade Vicente Pallotti, Santa Maria. 82p.

Riet-Correa B., Castro M.B., Lemos R.A., Riet-Correa G., Mustafa V. \& RietCorrea F. 2011b. Brachiaria spp. poisoning of ruminants in Brazil. Pesq. Vet. Bras. 31(3):183-192.

Riet-Correa F., Carvalho K.S., Dantas A.F.M. \& Medeiros R.M.T. 2011c. Spontaneous acute poisoning by Crotalaria retusa in sheep and biological control of this plant with sheep. Toxicon 58:606-609.

Rissi D.R., Rech R.R., Pierezan F., Gabriel A.L., Trost M.E., Brun J.S., Kommers G.D. \& Barros C.S.L. 2007. Intoxicações por plantas e micotoxinas associadas a plantas em bovinos no Rio Grande do Sul: 461 casos. Pesq. Vet. Bras. 27:261-268.

Schild A.L. 2008. Comunicação pessoal (Universidade Federal de Pelotas, Pelotas, RS.E-mail: alschild@terra.com.br)

Schuster J.L. 1978. Poisonous plants management problems and control measures on U.S. rangelands, p.23-34. In: Keeler R., Van Kampen K.R. \& James L.F. (Eds), Effetcs of Poisonous Plants on Livestock. Acedemic Press, New York. 600p.

Silva I.P., Lira R.A., Barbosa R.R., Batista J.S. \& Soto-Blanco B. 2008. Intoxicação natural pelas folhas de Mascagnia rigida (Malpighiaceae) em ovinos. Arqs Inst. Biológioco, São Paulo, 75(2):229-233.

Soares M.P., Riet-Correa F., Mendes M.C., Rosa F.G. \& Carreira E.G. 2000. Controle biológico de Senecio spp. com pastoreio de ovinos. Segunda Reunión Argentina de Patología Veterinaria, Corrientes, p.79-80.

Souza R.I.C., Riet-Correa F., Barbosa-Ferreira M., Brum K.B., Fernandes C.E. \& Lemos R.A.A. 2010. Intoxicação por Brachiaria spp. em bovinos no Mato Grosso do Sul. Pesq. Vet. Bras. 30(12):1036-1042.

Tokarnia C.H., Peixoto P.V. \& Döbereiner J. 1990. Poisonous plants affecting heart function of cattle in Brazil. Pesq. Vet. Bras. 10(1/2):1-10.

Tokarnia C.H., Döbereiner J., Dutra I.S., Brito I.S., Chagas B.R., França T.N. \& Brust L.A.G. 1999. Experimentos em bovinos com favas de Enterolobium contortisiliquum e E. timbouva para verificar propriedades fotossensibilizantes e/ou abortivas. Pesq. Vet. Bras. 19(1):39-45.

Tokarnia C.H., Döbereiner J. \& Peixoto P.V. 2002. Poisonous plants affecting livestock in Brazil. Toxicon 40:1635-1660.

Tokarnia C.H., Brito M.F., Barbosa J.D., Peixoto P.V. \& Döbereiner J. 2012. Plantas Tóxicas do Brasil. 2a ${ }^{a}$ ed. Editora Helianthus, Rio de Janeiro. 566p. 